\title{
Quantum tunnelling of the magnetisation in
}

\section{molecular nanomagnets}

R. Sessoli

Department of Chemistry, University of Florence and INSTM, 50019 Sesto Fiorentino, Italy

$T$

The tunnel effect represents the fascinating point where quantum mechanics meets the classical world. Even though not very easily observable, quantum tunnelling in charge transport is a well known phenomenon, now even exploited in devices, as in the Super Conducting Quantum Interference Device (SQUID). On the contrary the tunnelling phenomenon in magnetic materials is much less investigated. Most of the properties that make magnetic materials so important in our every-day life, i. e. magnetic hysteresis and related memory effect, do not reveal any macroscopic quantum effect. However, if the dimensions of the magnetic material are shrunk below the width of a domain wall, all the magnetic moments move coherently and the process of magnetisation reversal occurs by the overcoming of an energy barrier originated by the magnetic anisotropy of the material. This barrier can be crossed through a thermally activated process, but the presence of transverse anisotropy, or the application of a transverse field, can induce an underbarrier mechanism. The smaller the effective mass, here the magnetic moment that has to tunnel, the larger is the tunnel probability. Thus nanosized magnetic particles, comprising hundreds or thousands of coupled spins, are good candidate to show quantum tunnelling of the magnetisation (QTM), and even better are antiferromagnetic particles, where the magnetic moment is reduced to the uncompensated fraction on the surface. In the eighties the theory of QTM experienced a fast progression, however the experimental evidences of QTM were rather blurred. The major problem is

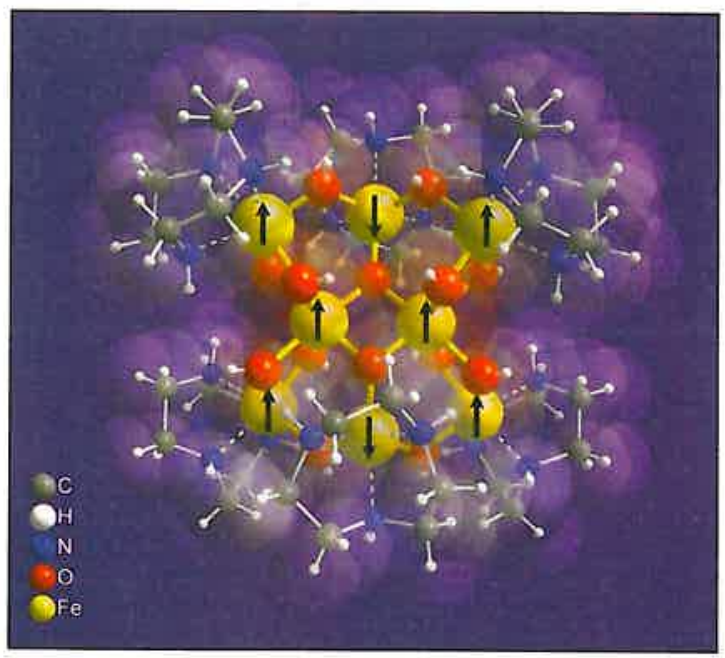

$\Delta$ Fig. 1: View of the structure of the Fe8 molecular cluster. The iron atoms (yellow) carry the magnetic moments that in the ground state are arranged to give $S=10$. The shadows around the cluster represent the actual dimensions of the atoms and give an idea of how the central magnetic core is surrounded by an organic shell. in fact due to the strong dependence of tunnelling on the axial and transverse magnetic anisotropy that, on their turn, are related to the dimensions of the nanoparticle. Ensembles of nanoparticles are unfortunately characterised by a distribution in size and shape.

\section{Molecular nanomagnets}

In the beginning of the nineties chemists, and in particular molecular chemists, entered into the game by starting to synthesise and investigate objects that can be seen as the "missing link" between the quantum word of paramagnetic metal centres and the classical one of magnetic particles. These new materials, known as molecular nanomagnets or as Single Molecule Magnets, are indeed clusters comprising a relatively small number of paramagnetic centres. These are usually paramagnetic transition metal ions, which interacts through bridging atoms, e.g. oxygen, or groups of atoms, e.g. the cyanide bridge. One example of this kind of molecules is the octanuclear iron cluster of formula $\left.\left[\mathrm{Fe}_{8} \mathrm{O}_{2}(\mathrm{OH})_{12} \text { (tacn }\right)_{6}\right] \mathrm{Br}_{8}$, abbreviated from here on as $\mathrm{Fe} 8$, whose structure is shown in Figure 1. The coordination sites of the iron atoms are saturated by the organic ligand tacn=triazacyclonane, which provides a hydrophobic shell preventing the growth of the metal hydroxide-oxide core to an extended lattice. The major advantage of the molecular approach, compared to the coating of nanosized particles, is that the clusters are arranged in a crystal structure and, in the most favourable cases neglecting also crystal defects, all the clusters are identical, equally oriented and weakly interacting among themselves. Despite the fact that oxygen atoms very often mediate moderate antiferromagnetic interactions, the complex connectivity and the related spin topology leads in some cases to a large spin multiplicity of the ground state. The example of Figure 1, Fe8, possess a ground $\mathrm{S}=10$ spin state. In this case the individual spins are those of rather isotropic, $\mathrm{d}^{5}$, iron(III) ions, with no orbital contribution. Also in the case of more anisotropic metal ions, as manganese(III), the orbital contribution is quenched by the low symmetry. Its presence is however important in the magneto-crystalline anisotropy, which is commonly described using an effective spin Hamiltonian of the form:

$H=\mathrm{DS}_{\mathrm{z}}{ }^{2}+\mathrm{E}\left(\mathbf{S}_{\mathbf{x}}{ }^{2}-\mathbf{S}_{\mathbf{y}}{ }^{2}\right)+\mathrm{g} \mu_{\mathrm{B}} \mathrm{S} \cdot \mathrm{H}$

The first term is the axial anisotropy responsible for the energy barrier which separates "spin up" and "spin down" configurations as shown in Figure 2, where the states are labelled with the eigenvalue of $\mathrm{S}_{2}, \mathrm{M}$. The double well potential drawn in the figure is reminiscent of that observed in classical single domain particles. Here only 21 well defined levels are present but at low temperature the thermally activated reversal of the magnetisation follows an Arrhenius law and the characteristic time becomes macroscopically long. If it grows over the time needed to measure an hysteresis cycle, a remanent magnetisation appears, with a coercive field which increases on lowering the temperature. The

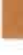


hysteresis is not related to a cooperative phenomenon but is a property of the isolated cluster that behaves like a magnet, a Single Molecule Magnet.[1] In Figure 3 is reported the hysteresis loop of Mn12-acetate, the archetype of SMM, also characterised by $S=10$, whose well-known structure is also schematised in Figure 3. A large temperature dependent remanent magnetisation is observed and also almost equally spaced steps. This step-like hysteresis, discovered by the group of M. Sarachick in New York[2] and almost at the same time in Grenoble by the group of B. Barbara in collaboration with the group of D. Gatteschi in Florence, [3] is now considered the fingerprint of the tunnelling of the magnetisation and the reason is immediately clear by looking at Figure 2. The transverse terms in the spin Hamiltonian do not commute with the axial term at the origin of the barrier and admix the left and right well localised states, thus allowing an under-barrier mechanism. The extent of this delocalisation is larger in the states close to the top of the barrier and maximum when the unperturbed levels on the opposite sites of the barrier are degenerate. In zero field (see Figure 2) all the pairs coincide in energy and this corresponds to the most favourable condition to observe tunnelling. Depending on the temperature and the thermal population of the levels, tunnelling between the ground states or levels higher in the barrier can compete with the thermally activated mechanism, and a short-cut of the barrier occurs with a significant acceleration of the magnetic relaxation. When a longitudinal field is applied the $\pm \mathrm{M}$ pairs are not in coincidence any more and tunnelling is suppressed, as in Figure $2 \mathrm{~b}$, except for critical values of $\mathrm{H}_{z}$ for which the levels are brought in resonance again. According to equation (1) pairs of level are in resonance for $\mathrm{H}_{z}=\mathrm{nD} / \mathrm{g} \mu_{\mathrm{B}}$, with $\mathrm{n}$ integer, as clearly seen in Figure 4. Accelerations of the relaxation due to tunnelling occur at these fields,
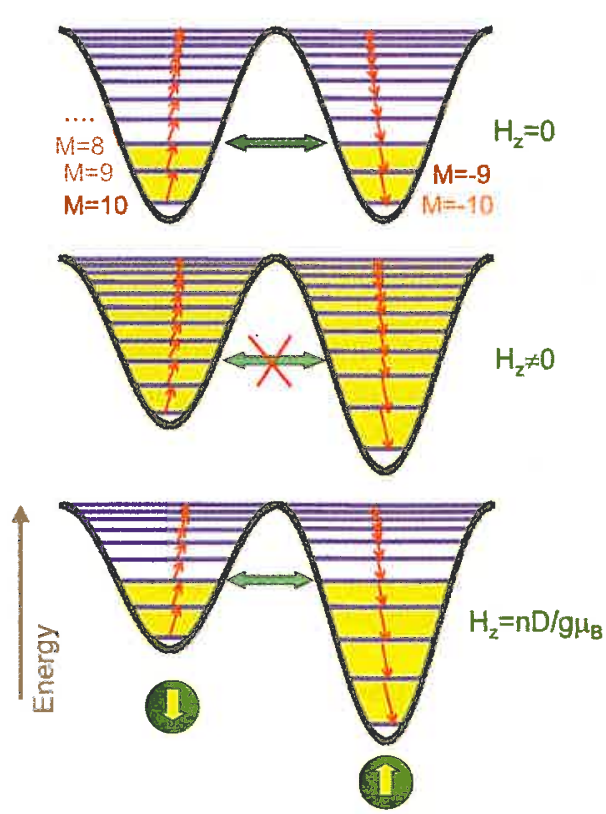

$\Delta$ Fig. 2: Energy levels of the $S=10$ spin manifold split by an axial anisotropy (top). Overcoming of the barrier can occur through a thermal activation or through a tunnel mechanism involving the ground doublet or thermally excited states. When an axial field is applied the levels on the opposite sides of the barrier are no more in coincidence (b) and tunnelling is suppressed unless specific values of the field are reached $(c)$.

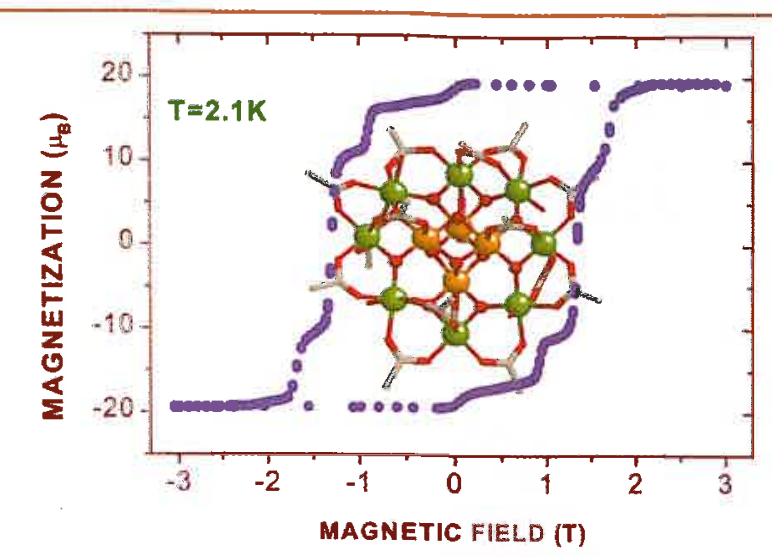

- Fig. 3: Stepped hysteresis cycle of the molecular nanomagnet Mn12-acetate, whose structure is also reported.

which correspond to the steps in the hysteresis curve. This mechanism was indeed first proposed by M.A. Novak and this author at the conference on "Macroscopic Quantum Tunneling of the Magnetization" held in 1994 close to Grenoble, where for the first time chemists introduced these novel materials to the physics community.[4]

Going back to Figure 2, a transverse field can be the source of the tunnelling, but when at low temperature pure tunnelling between the ground doublet occurs, a trivial calculation shows that a moderate field is not efficient enough, as it acts at the (2S) ${ }^{\text {th }}$ order of perturbation only. A transverse anisotropy is however always present, of second order in the absence of any symmetry as in (1), or of higher order, depending on the symmetry manifold of the molecule.

\section{Topological interference}

Intuitively one would expect that the application of a transverse field would anyhow enhance the tunnel process, but this is not the case. By exploiting arrays of micro SQUIDs it has been possible to monitor the reversal of the magnetisation by sweeping the longitudinal field back and forth on a tunnel resonance, which is actually an avoided level-crossing between two levels, as shown in Figure 4. The probability of reversing the magnetic moment depends on the sweeping rate and on the tunnel splitting $\Delta$, i.e. the gap at the avoided level-crossing, according to the Landau-ZenerStückelberg formula:

$$
P_{M, M^{\prime}}=1-\exp \left[-\frac{\pi \Delta_{M, M^{\prime}}^{2}}{2 h g \mu_{B}\left|M-M^{\prime}\right| \mu_{O} d H / d t}\right]
$$

where $\mathrm{dH} / \mathrm{dt}$ is the field sweeping rate. The tunnel splitting $\Delta$ is then extracted from (2) knowing $P$ by the fraction of molecules that has reversed magnetisation. The measurements performed on Fe8 in the pure tunnelling regime at very low temperature, with the procedure repeated for increasing transverse static fields applied along the axis of hard magnetisation, have shown the results reported in Figure 5. If the axial field is swept back and forth around zero the tunnelling splitting investigated is the one between the ground $M= \pm 10$ states. In this case the tunnelling is larger for zero transverse field, decreases to a very small value at ca. $0.2 \mathrm{~T}$ showing an almost complete quenching, and then increases again. An oscillatory behaviour is seen with an almost 
constant period in the whole investigated region.[5] On the contrary, if the transverse field is applied along the intermediate axis of magnetisation, the tunnel splitting increases steadily The oscillatory behaviour has the semi-classical physical description that is schematised in Figure 6. The sphere represents the continuum of orientation that a semi-classical spin $S$ can assume; $Z$ corresponds to the easy axis of magnetisation, while $\mathrm{X}$ and $\mathrm{Y}$ are the hard and intermediate ones, respectively. Let's assume that a static transverse field is applied along $\mathrm{X}$. The system can be prepared in such a way that $S$ is pointing toward $A$ by applying also a magnetic field along $+Z$. Then the longitudinal field is removed and the two orientation $A$ and $B$ becomes equally probable. An ensemble of molecules will show initially a net magnetisation pointing toward $+\mathrm{Z}$ but at the equilibrium half of the molecules must be pointing their spin toward $\mathrm{B}$. As the $\mathrm{X}$ direction is the less favourite one the spin will tend to rotate in a plane which is parallel to the $Y Z$ plane. The tunnelling transition from $A$ to $B$ on the sphere of Figure 6 can therefore occur along two equivalent paths, one clockwise and the other anti-clockwise. The two paths can interfere destructively leading to the quench of the tunnel splitting experimentally observed. The topological nature of the interference, even if in the spin space, has suggested for this phenomenon the name of magnetic Berry phase effect. The destructive interference occurs when the area highlighted in Figure 6 is equal to $\mathrm{k} \pi / \mathrm{S}$, with $\mathrm{k}$ an odd integer. This argument was presented by A. Garg some years before its experimental realisation, [6] when a large but well defined spin was still considered a text-book assumption. If we limit our analysis to the second order transverse anisotropy reported in (1) the quenching occurs when the transverse field assumes the values:

$$
H_{x}=\frac{2 n+1}{g \mu_{B}} \sqrt{E(E+D}
$$

where $\mathrm{n}$ is an integer. It is important to stress that at these fields an exact degeneration not related to the Kramers theorem occurs and this is an exception to the general rule that "levels repel each other". This situation is so exceptional that is sometime described as "diabolic". In this case the number of observed "diabolic fields" with quenching of the tunnel splitting is 10 on each field sign. This number corresponds also to how many times the perturbation induced by the $\mathrm{E}$ term in (3) must be applied to connect the two states involving the tunnelling. A numerical diagonalisation of the spin-Hamiltonian matrix allows one also to take into account different orientations of the transverse field in the XY plane. However one can immediately visualise that, when the transverse field is applied along the $Y$ direction, one tunnel pathway is favourable and no destructive interference is possible, as indeed is experimentally observed.

If the experiment is repeated by sweeping the longitudinal field around a value which corresponds for instance to the $M=10 \rightarrow M^{\prime}=-9$ resonance, thus $H_{z}^{0}=D / g \mu_{B}$, the transverse field dependence of the oscillation is completely different, as shown in Figure 5. Where maxima in tunnel splitting were previously present, minima are now observed, and viceversa. Now the two states are connected by an odd number of steps, exactly as in the case of half-integer spin states. The parity effect related to the time-reversal suggests that the tunnelling must be quenched in zero transverse field as experimentally observed. When the field is swept around $\mathrm{H}_{\mathrm{z}}{ }^{0}=2 \mathrm{D} / \mathrm{g} \mu_{\mathrm{B}}$, bringing $\mathrm{M}=10$ and $\mathrm{M}^{\prime}=-8$ in coincidence the oscillations have the same phase as for the $M=10 \rightarrow M^{\prime}=10$, as shown in Figure 5 . By simply changing the

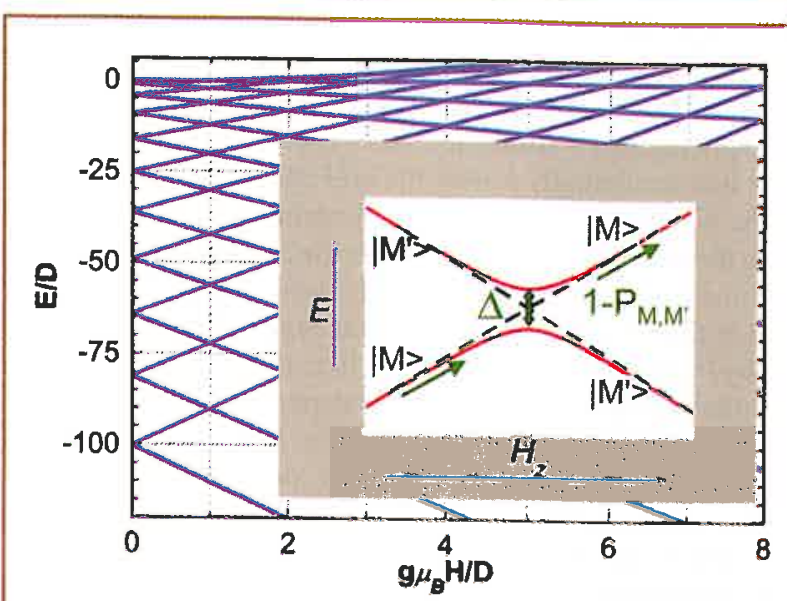

Alg. 4: Splitting of the $S=10$ manifold due to the application of a longitudinal field. In the inset an anticrossing is enlarged. $P$ represents the probability of reversing the magnetic moment. $\Delta$ is the tunnel splitting.

value of the field it is possible to modify the parity of the system.

All these experiments have provided elegant evidences of the tunnel mechanism in nanomagnets, and for these results the principal investigators (B. Barbara, J. Friedman, D. Gatteschi, W. Wernsdorfer and this author) have been awarded with the 2002 Agilent Technologies Europhysics Prize. Many other scientists have contributed to shed light on this new phenomenon by performing experiments that range from neutron spectroscopy to magnetic resonance, from muon spectroscopy to specific-heat measurements. Parallel to the experimental work, the contributions of many theoreticians have turned this field to what J.Villain has called "a school of physics".

\section{The role of the environment}

The story is however more complex than how it has been depicted up to now. In fact, it is sufficient to note the order of magnitude of the tunnel splitting in Figure 5 to immediately realise that some other mechanisms must be active to promote tunnelling. A tunnel splitting in the ground $M= \pm 10$ doublet of the order of $10^{-7} \mathrm{~K}$ requires that any bias field is smaller than $10^{-8} \mathrm{~T}$. Otherwise the level degeneracy, within the tunnel splitting, is removed and the admixing of the states is suppressed. Such a narrow tunnelling resonance could hardly be observed and indeed the experiments reveal tunnelling resonances, which are order of magnitude broader than this value. The clusters are not isolated objects but experience the dipolar field of the surrounding clusters in the crystal and a distribution of internal field is therefore expected. The origin of this field is the magnetisation of the molecules themselves, which is completely frozen at very low temperature if tunnelling does not occur. With a distribution width of the order of $2 \times 10^{-2} \mathrm{~T}$ and an intrinsic width of $10^{-8} \mathrm{~T}$ tunnelling should be a very rare event, unless the external field is swept over the entire distribution, as is the case for the Landau-Zener-Stückelberg type of experiment.

Prokof'ev and Stamp have proposed that at very low temperature tunnelling between the $\pm S$ pair can only occur if the fluctuating nuclear magnetic field is taken into account.[7] This fluctuating field has the effect of bringing in resonance the couple of levels where tunnelling is expected to occur. The reversal of the magnetic moment of a molecule inside the crystal modifies the dipolar field of the neighbouring sites and other molecules are 
thus brought close to the resonance condition where the fluctuating hyperfine field can be efficient again in promoting tunnelling. A pictorial representation of the phenomenon could be a box of small spheres and a small hole. If everything is static only the sphere that accidentally is over the hole can exit the box. However if the box is gently shaken, the hyperfine field, more spheres among the ones close to the hole, have the chance to go through it. As soon as a sphere exits the box the hole moves to a different region of the box and here the process restart. The theory developed by Prokof'ev and Stamp predicts that at short times the relaxation shows a square root time dependence of the decay of the remnant magnetisation, that has been indeed experimentally observed and also reproduced by using Montecarlo simulations. Transverse internal fields, of dipolar or nuclear origin, are also responsible for symmetry forbidden transitions.

The distribution of tunnel splitting can be investigated experimentally by using a technique, which is well known in single molecule spectroscopy: the hole digging. The results of these experiments have revealed that at high temperature the line has a lorentzian shape and no holes can be dug. The linewidth is dominated by the homogeneous broadening induced by the larger tunnel splitting between levels, which are higher in the barrier. On the contrary at very low temperature a hole can be burnt and the intrinsic width of the hole is strongly dependent on the hyperfine field generated by the nuclei. This has been shown by measuring also two isotopically modified samples of the Fe8 molecular nanomagnets. In particular the substitution of the non-magnetic ${ }^{56} \mathrm{Fe}$ nuclei with ${ }^{57} \mathrm{Fe}$ possessing $\mathrm{I}=1 / 2$ increases the hyperfine field, which, on the contrary, can be reduced by replacing hydrogen with deuterium. Unfortunately the large contribution due to the nuclei of ${ }^{14} \mathrm{~N}$ in the tacn ligand cannot be modified. Anyhow a significant trend in the linewidth of the hole dug in the tunnelling resonance is observed. The ${ }^{57} \mathrm{Fe}$ isotope has the largest linewidth and the fastest relaxation and the deuterated sample shows the narrowest resonance and the slowest relaxation.

The role of nuclear magnetism is even more important in another class of materials of different origin but with many analogies with molecular nanomagnets. B. Barbara and co-workers have shown that rare earth anisotropic ions, like $\mathrm{Ho}^{3+}$, in the diamagnetic host lattice, $\mathrm{LiYF}_{4}$, can experience an energy barrier which hampers the magnetisation reversal. The interaction of the electronic ground doublet with the $\mathrm{I}=7 / 2$ nuclear magnetic moment of the $\mathrm{Ho}^{3+}$ ions gives rises to a stepped hysteresis where the steps correspond to avoided level crossings within the 16 manifold of the hyperfine splitted doublet.

The detailed investigation of the dynamics of the magnetisation of molecular nanomagnets has also revealed other important effects. Recently W. Wernsdorfer, in collaboration with American chemists G. Christou and D. N. Hendrickson, has investigated a wide range of tetranuclear manganese clusters and in one of them the clusters are coupled in pairs by hydrogen-bonds. The effects of these interactions on the tunnelling are well evident. The quantum transition in zero longitudinal field, which corresponds to the simultaneous tunnelling of both spins of the pair, is suppressed and other transitions are observed that are assigned to spin cross-relaxation. [8]

Crystal defects or disorder effects in Mn12-acetate leading to deviations from the tetragonal symmetry have also been the subjects of recent theoretical and experimental studies. This could appear as a contradiction, suggesting that the systems are not so ideal as initially proposed. On the contrary only "clean" systems and "clean" experimental results can allow an investigation of the nature of the defects by looking at their effects on the quantum dynamics of the magnetisation.

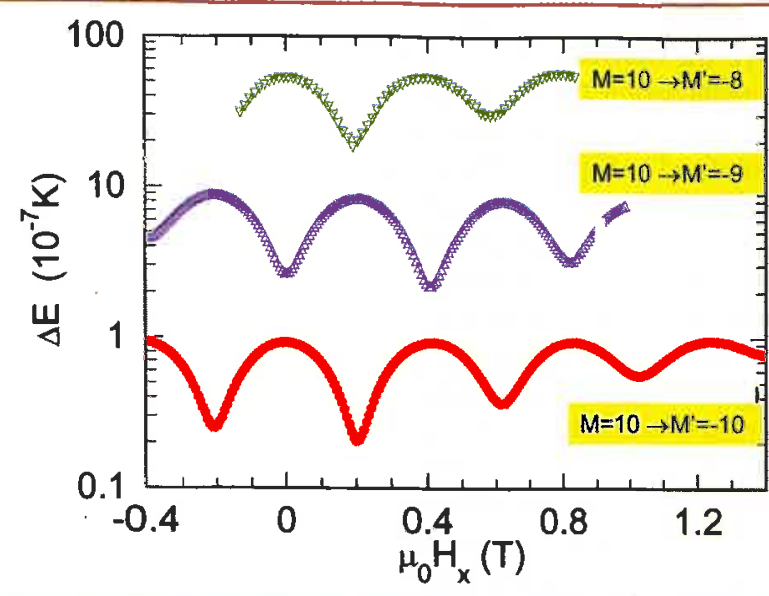

A Fig. 5: Transverse field dependence of the tunnel splitting in Fe 8 measured at $80 \mathrm{mK}$. The different curves are for the anticrossings involving different pairs of levels. A phase shift is observed depending on the parity of the $M \rightarrow M^{\prime}$ transition.

\section{Possible applications}

Up to now we have attended "the school of physics" provided by these elegant applications of quantum mechanics but the Agilent Technologies Europhysics Prize awards fundamental works "leading to advances in the fields of electronic, electrical and materials engineering". How can our research fulfil these expectations? It is trivial to say that in the materials we have investigated each molecule behaves as a magnetic memory unit, but it is hard to conceive a PC hard disk made of Mn12-acetate. First of all we have to learn how to address a single molecule. We have seen that the effects of the environment are far from negligible and to monitor them chemists are now going beyond the single crystal samples and are organising the molecules in a controlled way, for instance attaching them on the surface of a conductor. The topologic interference effect, if observed in other magnetic materials, could be exploited to achieve a better control of the magnetisation process, which is very important to obtain stable and easily writeable mag-

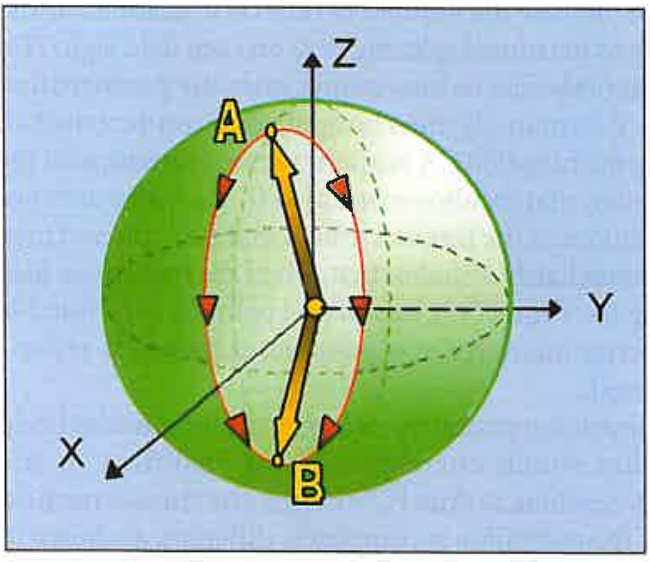

A Fig. 6: Unit sphere showing the reversal of a classical spin. The $X, Y$, and $Z$ axis are the hard, intermediate, and easy axis, respectively. The spins point toward $\pm Z$ in zero field. Two paths, clock and anti-clock wise, connect the two degenerate minima $A$ and $B$ (whose position depends on the transverse field). The two paths give destructive interference whenever the highlighted area is $\mathrm{k} \pi / \mathrm{S}$, with $\mathrm{k}$ an odd integer. 
netic supports. Leuenberger and Loss have also tried to visualise the exploitation of molecular nanomagnets for quantum computing [9]but an experiment based on their ideas has not yet been realised. We have first to learn to control the tunnelling process by using an electromagnetic field. Chudnovsky and Garanin have even proposed the existence of a super-radiance phenomenon if the tunnelling transition is swept rapidly enough to achieve the population inversion and all the spins tunnel in a coherent way. Some experiments have been recently performed, on $\mathrm{Mn} 12$ acetate crystals again.

The story is far from coming to an end, also because there is no limit to the ingenuity and fantasy of chemists. They continue in designing and "creating" novel molecules that range from one dimensional magnetic systems closed on themselves to provide magnetic rings of various size, to buckyball magnetic clusters that seem to have lost most of their quantum nature.

We have reached the end of this short overview where a chemist has been invited to write in a physical magazine. This fact reminds me of a dialogue between Primo Levi, a famous writer but also an enthusiastic chemist, and the Italian physicist Tullio Regge. I find the words of Regge "The universe must be infinite to realise everything is permitted, because what is permitted is also compelled to be" very adequate to describe the role that chemists have played in this story. Chemists have just condensed more of these possibilities in small molecules. We have then served to physicists these little toys to observe hidden phenomena of quantum mechanics that, after all, had to be observed.

\section{Acknowledgments}

I thank Bernard Barbara, Jonathan Friedman, Dante Gatteschi and Wolfgang Wernsdorfer who have enthusiastically pushed me to report here my personal view of our awarded research. I thank also the many colleagues, strongly involved in this research, but not quoted here for the lack of space, whose contributions have been of fundamental importance for the development of this field.

\section{References}

[1] R. Sessoli, D. Gatteschi, A. Caneschi, and M. A. Novak, Nature (London) 365, 141 (1993).

[2] J. R. Friedman, M. P. Sarachik, J. Tejada, and R. Ziolo, Phys. Rev. Lett. 76, 3830 (1996).

[3] L. Thomas, F. Lionti, R. Ballou, D. Gatteschi, R. Sessoli, and B. Barbara, Nature (London) 383, 145 (1996).

[4] Quantum Tunneling of Magnetization - QTM'94 L. Gunther and B. Barbara (Kluwer, Dordrecht, 1995), p. 171.

[5] W. Wernsdorfer and R. Sessoli, Science 284, 133 (1999).

[6] A. Garg, Europhys. Lett. 22, 205 (1993).

[7] N.V. Prokof'ev and P. C. E. Stamp, Phys. Rev. Lett. 80, 5794 (1998).

[8] W. Wernsdorfer, N. Allaga-Alcalde, D. N. Hendrickson, and G. Christou, Nature 416, 406 (2002).

[9] M. N. Leuenberger and D. Loss, Nature 410, 789 (2001).

\section{Crowd control}

T. Vicsek

Department of Biological Physics, Eötvös University, Pázmány p. Stny 1A, H-1117 Budapest, Hungary

$T$ he application of ideas, methods and results of statistical physics to a wide range of phenomena occurring outside of the realm of the non-living world has gained a great momentum recently. Among many others, examples include the studies of various group activities of people from the physicist's viewpoint. Here, I shall give a partial account of some of the new investigations in this direction, involving the interpretation of such collective human activities as group motion and synchronisation.

On the small scale side of the size/complexity spectrum, in the world of atoms and molecules collective behaviour is also considered to be an important aspect of the observed . Furthermore, there are articles on collectively migrating bacteria, insects or birds and additional interesting results are published on phenomena in which groups of various organisms or non-living objects synchronise their signals or motion. This is the natural scientist's aspect of how many objects behave together. However, if you search for a collective behaviour related item with your web browser most of the texts popping up will be concerned with group activities of humans including riots, fashion or panics.

What is common in these seemingly diverse phenomena involving interpretations ranging from social psychology to statistical physics? The answer is that they happen in systems consisting of many similar units interacting in a relatively well defined manner. These interactions can be simple (attraction/repulsion) or more complex (combinations of simple interactions) and may take place between neighbours in space or on a specific underlying network. Under some conditions, in such systems various kinds of transitions occur; during these transitions the objects (particles, organisms or even robots) adopt a pattern of behaviour which is nearly completely determined by the collective effects due to the presence of all of the other objects in the system.

Take ferromagnets as an example. These materials can undergo spontaneous magnetisation, in effect because they are made up of a host of "tiny magnets". At relatively high temperatures, these magnets cannot align with each other and the resulting magnetisation is zero. But at a critical temperature the tendency to adopt a common direction suddenly, but continuously takes over from the effects of fluctuations. So, most of the small magnets, assisting each other in a collective manner, point in the same direction, and magnetisation (order) spontaneously appears. Similarly, a group of feeding pigeons randomly oriented on the ground will order themselves into a uniform flock while flying away after a big disturbance.

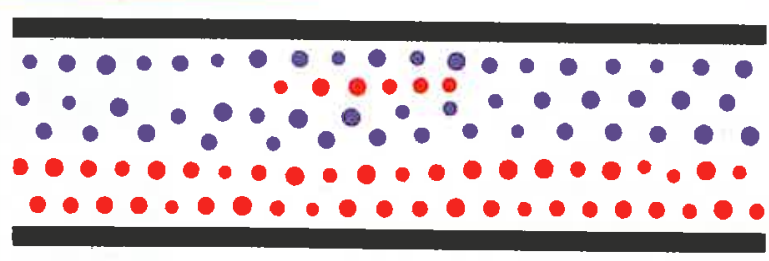

A Fig. 1: Spontaneous formation of lanes in a system of oppositely driven particles with repulsive interactions. Similar segregation of pedestrian flows can be observed, e,g., along wide corridors. 